\title{
Photonic band gap in the triangular lattice of Bose-Einstein-condensate vortices
}

\author{
M. E. Taşgın, ${ }^{1}$ Ö. E. Müstecaplığlu, ${ }^{2}$ and M. Ö. Oktel ${ }^{1}$ \\ ${ }^{1}$ Department of Physics, Bilkent University, 06800 Bilkent, Ankara, Turkey \\ ${ }^{2}$ Department of Physics, Koç University, 34450 Sartyer, Istanbul, Turkey
}

(Received 26 March 2007; published 27 June 2007)

\begin{abstract}
We investigate the photonic bands of an atomic Bose-Einstein condensate with a triangular vortex lattice. Index contrast between the vortex cores and the bulk of the condensate is achieved through the enhancement of the index via atomic coherence. The frequency-dependent dielectric function is used in the calculations of the bands, resulting in photonic band gap widths of a few megahertz.
\end{abstract}

DOI: 10.1103/PhysRevA.75.063627

PACS number(s): 03.75.Lm, 42.50.Gy, 42.70.Qs, 74.25.Qt

\section{INTRODUCTION}

A rotating Bose-Einstein condensate (BEC) manifests the formation of vortices after a critical rotation frequency. Furthermore, the constituent vortices exhibit a periodic structure, which is generally a triangular lattice [1-4]. Even near the borders of the condensate, lattice distortion is small [4]. From the theoretical point of view, a rapidly rotating BEC can be treated analytically, and the density is found to be the product of a slowly varying function and a periodic function $[3,4]$.

Usually the imaging of the vortices is carried out during the ballistic expansion of the condensate [1,2,5-7]. An in situ imaging was accomplished recently, by visualizing the twodimensional (2D) image of the lattice along the rotation axis, while the condensate was in the trap [8].

In the BEC experiments, the rotation frequency is not directly measured, but deduced from the change in the aspect ratio of the cloud $[1,2,5-7]$. However, we recently proposed a method based on the reflection through directional pseudo photonic band gaps [9]. Photonic band gaps in a triangular vortex lattice are obtained through the enhancement of the refractive index via quantum coherence $[10,11]$, so that sufficient index difference is generated between the vortex cores and the bulk of the condensate. Without an index enhancement scheme, the usual rotating BEC with a vortex lattice cannot exhibit high enough index contrast to obtain photonic band gaps. BECs are rather dilute, and, being an atomic gaseous medium, they exhibit dispersion only in highly absorptive regimes. When the ground and excited states are coupled to other auxiliary levels, however, absorption in the resonant transition of the probe beam can be canceled. This is due to the quantum interference of various absorption paths. This way, one can benefit from the large dispersion at an atomic resonance, without absorption.

Utilizing the index enhancement scheme, a triangular lattice of BEC vortices can generate both directional and complete photonic band gaps. Directional pseudogaps are also called partial or stop gaps [12]. Radiation cannot propagate in certain directions determined by these pseudogaps, but can in others. The rotation frequency of the condensate can be measured from the chopping in the reflected or transmitted probe beam at a directional photonic band gap.

In a previous study [9], we demonstrated the presence of a photonic band gap within the frequency window of index enhancement. In this paper, we discuss the photonic band structure for the full frequency regime, extending our work beyond the index enhancement window.

Although our general examination simply verifies the existence of the band gap within the index enhancement window, the electric susceptibility becomes a complex-valued function of frequency beyond this region, for which definition of photonic band gaps is not straightforward. In the particular index enhancement scheme we consider here, there are absorption and gain regimes, where we have found no photonic band gaps. This allows for selective stoppage of the probe pulse among the other electromagnetic fields that are in use for trapping the condensate atoms and for index enhancement schemes.

Despite the dense literature on photonic crystals, studies of photonic energy bands which take complex, frequencydependent dielectric constants into account are rather sparse. Few recent studies investigate materials with small absorption [13] and low filling fractions of the dispersive and absorptive component [14-16]. More recent works go beyond these limitations [17-19]. These efforts focus on understanding the properties of photonic crystals fabricated from metallic materials, which can have large complete photonic band gaps in the visible region of the electromagnetic spectrum. Drude-like models of metallic components within a dielectric host are used in the modeling of such crystals. Absorption is of negligible importance within the transparency window of the Drude model, which is about half of the plasma frequency. Small, yet realistic, amounts of absorption hardly change the band structure [15]. In the regions of appreciable absorption, however, there may be no band gaps [18]. Our results for a rotating atomic BEC are in agreement with these results for photonic crystals of metallic materials. We note that, quite recently, broadband absorptive properties of metallic photonic crystals are found to be advantageous for various applications [20]. Similarly, the gain regime is important for understanding lasing properties of photonic crystals [21]. Our suggested index enhancement schemes for rotating BECs offer absorptive as well as gain regimes beyond the index enhancement window [10], associated with lasing without inversion [11].

The understanding of photonic crystals with complex dielectric constants is not trivial, due to the lack of a welldefined group velocity for complex energy bands [22]. In this paper, we limit ourselves to the determination of the band structure, and do not discuss the details of beam propagation beyond the existence of the band gaps. 


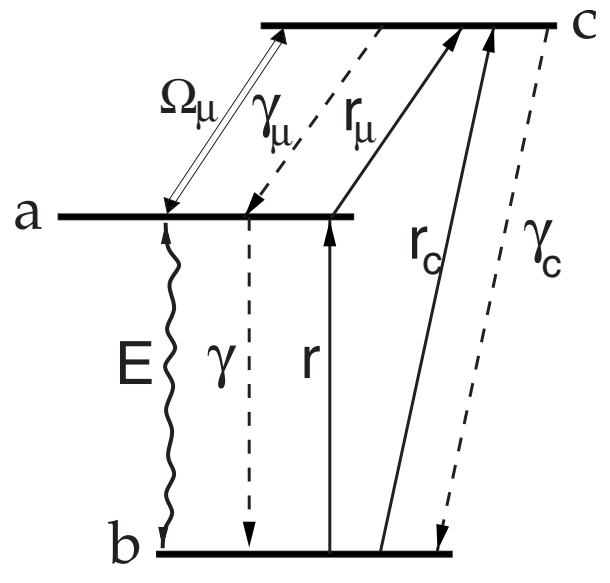

FIG. 1. Upper-level microwave scheme for index enhancement [10]. Upper two levels $a$ and $c$ are coupled via a strong microwave field of Rabi frequency $\Omega_{\mu}$. Weak probe field $E$ of optical frequency $\omega$ is coupled to levels $a$ and $b$. Decay $(\gamma)$ and pump $(r)$ rates are indicated.

The paper is arranged as follows. In Sec. II, we give an overview of the upper-level microwave scheme, which leads to the index enhancement with vanishing absorption. We introduce the system parameters and the resulting dielectric susceptibility. In Sec. III, we obtain the matrix equations from the master equation of the photonic crystals and illustrate the method of solution for the case of a frequencydependent, complex dielectric function. In Sec. IV, we present the resulting photonic bands for two different lattice parameters, and then discuss the properties of the photonic bands. Section V is a summary of our results.

\section{DIELECTRIC FUNCTION OF THE VORTEX LATTICE}

In this section, we describe the atomic coherence and path interference effects leading to a high index of refraction with vanishing absorption. We review the derivations of Ref. [10] in a compact form, and describe the physics of the system. We calculate the real and imaginary parts of the dielectric susceptibility.

There are various index enhancement schemes [10]. Among them, we specifically consider the upper-level microwave scheme as it leads to strong index contrast, although the Raman scheme may also be useful due to its wider frequency window for index enhancement [10]. The corresponding level diagram for the upper-level microwave scheme is shown in Fig. 1. A weak optical probe field $E$ of frequency $\omega$ is coupled to the two levels $a$ and $b$ through the electric dipole interaction. A third level $c$ is also coupled to level $a$ via a strong resonant microwave field of Rabi frequency $\Omega_{\mu}$. Coupling level $a$ to level $c$ allows for different possible paths of absorption. Destructive quantum interference of these paths may cancel the absorption of the probe field at a certain frequency [11]. At the same time, a high refractive index can be generated by maintaining some population in level $a$ to ensure high dipole moments for levels $a$ and $b$. For that aim, indirect pump mechanisms are introduced. The parameters $r_{\mu}, r$, and $r_{c}$ are the pump rates from level $a$ to $c, b$ to $a$, and $b$ to $c$, respectively.
In Fig. $1, \gamma_{\mu}, \gamma$, and $\gamma_{c}$ denote the decay rates (inverse lifetimes) of levels $c$ to $a, a$ to $b$, and $c$ to $b$, respectively, due to collisions and radiation. We can consider $\gamma_{\mu}, \gamma_{c} \ll \gamma$, as $\gamma_{c}$ and $\gamma_{\mu}$ are for dipole-forbidden and microwave transitions, respectively.

Following Ref. [10], we set $r_{\mu}=r=0$, and choose $r_{c}=\Omega_{\mu}$ $=\gamma$. We neglect $\gamma_{\mu}$ and $\gamma_{c}$. The decoherence rates between the denoted levels become $\gamma_{a b}=\gamma, \gamma_{a c}=\gamma / 2$, and $\gamma_{c b}=\gamma / 2$.

In that case, the frequency-dependent electric susceptibility $\chi(\omega)=\chi^{\prime}(\omega)+i \chi^{\prime \prime}(\omega)$ is a complex function of frequency, with its real and imaginary parts $\chi^{\prime \prime}$ given by [10]

$$
\begin{aligned}
& \chi^{\prime}(\varpi)=\frac{12 N \lambda^{3}}{13 \pi^{2}} \frac{\varpi}{9-3 \varpi^{2}+4 \varpi^{4}}, \\
& \chi^{\prime \prime}(\varpi)=-\frac{3 N \lambda^{3}}{13 \pi^{2}} \frac{-3+2 \varpi^{2}}{9-3 \varpi^{2}+4 \varpi^{4}},
\end{aligned}
$$

where $N$ is the number density of atoms and $\lambda$ is the wavelength of the optical transition $a \rightarrow b$. We define a dimensionless frequency

$$
\varpi=\left(\omega-\omega_{a b}\right) / \gamma,
$$

centered at the resonance frequency $\omega_{a b}$ and scaled with the decay rate of atomic coherence.

The susceptibilities given by Eqs. (1) and (2) are for a dilute condensate. In the case of a dense condensate, the first correction to the susceptibility is equivalent to a local field correction [23] in the form

$$
\chi_{\mathrm{loc}}(\varpi)=\frac{\chi(\varpi)}{1-\chi(\varpi) / 3} .
$$

The real and imaginary parts of the corresponding dielectric function,

$$
\epsilon_{\mathrm{loc}}(\varpi)=1+\chi_{\mathrm{loc}}(\varpi)
$$

are plotted in Fig. 2 for rubidium-87 gas. The vertical line indicates the enhancement of the polarization at the frequency of vanishing absorption. Here, we define $\Omega_{0}=2.37 \times 10^{15} \mathrm{~Hz}$ as the frequency at which absorption is zero. We also define $\varpi_{0}=\left(\Omega_{0}-\omega_{a b}\right) / \gamma \simeq 1.22$ as the corresponding value of the scaled and shifted frequency $\varpi$. We employ these definitions throughout the paper.

Isotopes of alkali metals are typically used in the BEC experiments, and we specifically consider the energy levels of rubidium. The fine-structure energy levels of rubidium, which correspond to $b, a$, and $c$ levels of Fig. 1, are $5 s_{1 / 2}$, $5 p_{1 / 2}$, and $6 s_{1 / 2}$, respectively. The wavelengths of the $a-b$ and $a-c$ transitions become $\lambda=794 \mathrm{~nm}$ and $\lambda_{\mu}=1.32 \mu \mathrm{m}$. The lifetime of the probe resonance level $\left(5 p_{1 / 2}\right)$ is $27 \mathrm{~ns}$, which corresponds to the decay rate $\gamma=2 \pi \times 6 \mathrm{MHz}$.

In the vicinity of the center of the condensate cloud, the dielectric function plotted for the peak density in Fig. 2 can be assumed. When vortices are present in this central region, however, their spatial profile will influence the dielectric function. Assuming a dilute thermal gas background at ultracold temperatures, index enhancement will be influential on the dense condensate only. The density of the condensate drops rapidly to zero at the vortex positions. The dielectric 

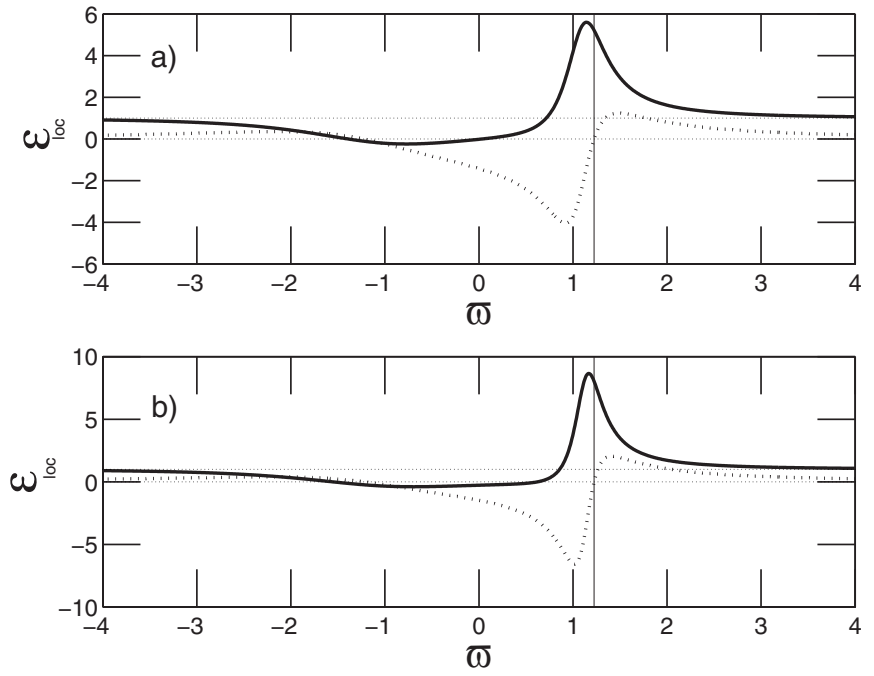

FIG. 2. Real (solid line) and imaginary (dotted line) parts of local dielectric function $\epsilon_{\mathrm{loc}}(\omega)$ as a function of scaled frequency $\varpi=\left(\omega-\omega_{a b}\right) / \gamma$, for the particle densities (a) $N=5.5 \times 10^{20} \mathrm{~m}^{-3}$ and (b) $6.6 \times 10^{20} \mathrm{~m}^{-3}$. Vertical solid line indicates the scaled enhancement frequency $\varpi_{0} \simeq 1.22$, where $\epsilon_{\mathrm{loc}}^{\prime \prime}(\varpi)$ vanishes. (a) $\epsilon=\epsilon_{\mathrm{loc}}\left(\varpi_{0}\right)$ $=5.2$ and (b) $\epsilon=\epsilon_{\mathrm{loc}}\left(\varpi_{0}\right)=8.0$.

constant within the vortex core can be supposed to be the same as that of vacuum, $\epsilon_{0}=1$. The width of the vortices is on the order of the coherence length of a condensate, which is given by $\xi=1 / \sqrt{8 \pi N a_{\mathrm{sc}}}$, where $a_{\mathrm{sc}}$ is the s-wave scattering length of the interatomic collisions. The typical value of the coherence length is a few hundred nanometers and smaller than the optical wavelength. Spatial modulations on the dielectric function can be introduced by $\epsilon=\epsilon_{0}+\left(\epsilon_{\mathrm{loc}}-\epsilon_{0}\right) \rho(\vec{r})$ so that

$$
\epsilon_{\mathrm{loc}}(\vec{r}, \varpi)=1+\rho(\vec{r}) \chi_{\mathrm{loc}}(\varpi) .
$$

Here $\rho(\vec{r})$ stands for the normalized spatial profile of a vortex, and $\vec{r}$ is the radial distance from the center of the vortex core. This model dielectric function (6) drops to the vacuum value at the cores of the vortices and recovers its bulk value in a few coherence lengths.

In the band calculations for a triangular lattice of vortices, we considered a hexagonal Wigner-Seitz unit cell, which contains a single vortex core at the center of the unit cell. We used Padé's analytical form, derived in Ref. [24], for the vortex density profile,

$$
\rho(r)=\frac{r^{2}\left(0.3437+0.0286 r^{2}\right)}{1+0.3333 r^{2}+0.0286 r^{4}},
$$

where $r$ is scaled with the coherence length $\xi$. This density behavior is valid in one unit cell. $\rho(r)$ becomes zero at the center and goes to 1 toward the edges of the unit hexagonal cell. We choose the lattice constant $a$ in terms of the coherence length to fix the filling factor of the vortices. We used two different values $a=10 \xi$ and $4.5 \xi$, in the computations.

Susceptibilities (1) and (2) are derived in the restricted condition of the microwave coupling strength $\Omega_{\mu}=\gamma$. In experiments, however, the strength of the laser is tunable in order to obtain different behaviors of the dielectric function.
Increasing $\Omega_{\mu}$ shifts the peak of $\chi^{\prime}$ away from zero detuning. So, zero absorption is obtained at a frequency greater than $\varpi=1.22$. However, the peak diminishes on being shifted. On the other hand, when $\Omega_{\mu}$ is decreased, the coherence between the levels $b$ and $c$ is reduced. This results in higher absorption. To obtain index enhancement at different frequencies by tuning $\Omega_{\mu}$, one has to compromise on the index contrast.

In the following sections, we discuss the propagation of the probe beam through a vortex lattice that has a dielectric function given by Eq. (6) and look for possible band gap formation about the index enhancement frequency.

\section{CALCULATION OF THE PHOTONIC BANDS}

The stable lattice type for a single-component rotating BEC is triangular $[1,2]$. The density profile is composed of vortices distributed periodically and an envelope density profile that decreases toward the edges of the trap. The envelope is a slowly varying function compared to the periodicity of the vortices. The radius of the cloud is much greater than the periodicity, such that there may be a few hundred vortices that are experimentally observable. Moreover, the distortion of the lattice near the edges of the condensate is small.

In our past work [9], we have numerically investigated the effects of the finite size and imperfections in the periodicity. We observed that the positions of the gaps are not strongly affected, despite the occurrence of extra scattering due to the smooth density envelope over the lattice.

Thus, in this paper, we consider an infinite homogeneous vortex lattice and concentrate on the effects of the frequency dependence of the dielectric function.

A two-dimensional photonic crystal supports only two polarization modes for the in-plane propagation of light [12]. If the magnetic field $\vec{H}$ is perpendicular to the plane of periodicity, this mode is called transverse electric (TE). In a TE mode the electric field $\vec{E}$ is perpendicular to the axis of the vortices. Similarly, the mode with $\vec{E}$ parallel to the vortex axis is called the transverse magnetic (TM) mode.

Let us first focus on the TE modes. We take the vortices to be aligned in the $\hat{z}$ direction, forming a periodic array in the $x-y$ plane. A generalized eigenvalue equation for $\vec{H} \| \hat{z}$,

$$
\vec{\nabla} \times\left(\frac{1}{\epsilon(\vec{r}, \omega)} \vec{\nabla} \times \vec{H}(\vec{r})\right)=\left(\frac{\omega}{c}\right)^{2} \vec{H}(\vec{r}),
$$

is derived by decoupling the Maxwell equations for $\vec{H}$, after the substitution $\vec{D}(\vec{r}, \omega)=\epsilon(\vec{r}, \omega) \vec{E}(\vec{r}, \omega)$ [15]. Unlike in the frequency-independent case, the differential operators on the left-hand side also depend on $\omega$. Moreover, the differential operator is not Hermitian because of the imaginary part of the dielectric function (6). This causes the eigenfrequencies to be complex. Since, in general, Eq. (8) is not analytically solvable, we determine the eigenfrequencies computationally by plane wave expansion.

Using the Bloch-Floquet theorem [25], the magnetic field can be expressed in terms of the reciprocal lattice vectors $\vec{G}$ as 


$$
\vec{H}=\sum_{\vec{G}} H_{\vec{G}} e^{i(\vec{k}+\vec{G}) \cdot \vec{r}} \hat{z} .
$$

Similarly, the inverse dielectric function is expanded as

$$
\frac{1}{\epsilon(\vec{r}, \omega)}=\sum_{\vec{G}^{\prime}} \varepsilon_{\vec{G}^{\prime}} e^{i \vec{G}^{\prime} \cdot \vec{r}},
$$

where the Fourier components $\varepsilon_{G^{\prime}}$ are

$$
\varepsilon_{G^{\prime}}(\omega)=\frac{1}{A} \int \frac{e^{-i \vec{G}^{\prime} \cdot \vec{r}}}{\epsilon(\vec{r}, \omega)} d^{2} \vec{r} .
$$

The integration is carried out over the Wigner-Seitz unit cell of area $A$.

We substitute the expansions (9) and (10) into the master equation (8), and obtain the expression

$$
\sum_{\vec{G}^{\prime}} \varepsilon_{\left(\vec{G}-\vec{G}^{\prime}\right)}(\omega) H_{G^{\prime}}\left[(\vec{k}+\vec{G}) \cdot\left(\vec{k}+\vec{G}^{\prime}\right)\right]=\left(\frac{\omega}{c}\right)^{2} H_{\vec{G}} .
$$

We note that, if the dielectric function were real, $\varepsilon_{\vec{G}-\vec{G}^{\prime}}^{*}$ $=\varepsilon_{G^{\prime}-\vec{G}}$, the matrix represented by Eq. (12) would be real. The eigenfrequencies would also be real. Moreover, due to inversion symmetry of the unit cell the $\epsilon_{\vec{G}}^{\overrightarrow{ }}$ 's would be real. However, the presence of a complex dielectric function destroys the Hermiticity. The eigenfrequencies are, in general, complex.

The real space basis vectors for a triangular lattice are $\vec{a}_{1}=a \hat{x}$ and $\vec{a}_{2}=a\left(\frac{1}{2} \hat{x}-\frac{\sqrt{3}}{2} \hat{y}\right)$. The corresponding reciprocal lattice basis vectors are $\vec{b}_{1}=k_{0}\left(\frac{\sqrt{3}}{2} \hat{x}-\frac{1}{2} \hat{y}\right)$ and $\vec{b}_{2}=k_{0} \hat{y}$, where the magnitude of both vectors is $k_{0}=(2 / \sqrt{3})(2 \pi / a)$. Any lattice point in the summation can be written as $\vec{G}=n_{1} \vec{b}_{1}+n_{2} \vec{b}_{2}$, where $n_{1}$ and $n_{2}$ are integers. We also denote the Fourier components of the inverse dielectric function as $\varepsilon_{\vec{G}} \equiv \varepsilon_{n_{1}, n_{2}}$.

For computational purposes, we limit the number of $\vec{G}$ vectors over which the summation will be carried out. We consider a parallelogram in the reciprocal space over which $n_{1}$ and $n_{2}$ run from $-N$ to $N$. $N$ is a positive integer. This gives a $(2 N+1)^{2} \times(2 N+1)^{2}$ matrix of elements

$$
\begin{aligned}
M_{i j}(\omega)= & \varepsilon_{\eta_{1}, \eta_{2}}(\omega)\left[\left(\vec{k}+n_{1} \vec{b}_{1}+n_{2} \vec{b}_{2}\right) \cdot\left(\vec{k}+n_{1}^{\prime} \vec{b}_{1}+n_{2}^{\prime} \vec{b}_{2}\right)\right] \\
& -\left(\frac{\omega}{c}\right)^{2} \delta_{i j},
\end{aligned}
$$

where the dependences of the indices are given by

$$
i=(2 N+1) n_{1}+n_{2} \text { and } j=(2 N+1) n_{1}^{\prime}+n_{2}^{\prime} .
$$

We use the notations

$$
\eta_{1}=n_{1}-n_{1}^{\prime} \quad \text { and } \quad \eta_{2}=n_{2}-n_{2}^{\prime},
$$

for which $\varepsilon_{\eta_{1}, \eta_{2}}(\omega)$ describes the Fourier component with the reciprocal wave vector $\vec{G}^{\prime}=\eta_{1} \vec{b}_{1}+\eta_{2} \vec{b}_{2}$ [Eq. (11)].

The solution of the master equation (8) simplifies to the determination of eigenfrequencies $\omega$ for each wave vector $\vec{k}$. All distinct values of $\omega$ are obtained by choosing $\vec{k}$ in the first Brillouin zone.
When the dielectric function is independent of $\omega$, the eigenfrequencies can be easily determined by straightforward matrix diagonalization [12]. However, the dependence of Fourier elements $\varepsilon_{\vec{G}^{\prime}}(\omega)$ on the frequency forces the calculations to be carried out by relying on the condition of vanishing determinant,

$$
\operatorname{det}(M)=0 .
$$

Numerical calculations are based on finding the zeros of the determinant of the matrix $\mathbf{M}$, as a function of $\vec{k}$ or $\omega$. The zeros of the complex function are computed using a leastsquares method. We have checked the convergence of the solutions using different initial points.

In the constant dielectric case, one chooses a $\vec{k}$ value as the input and determines the $\omega$ value. This is because $\omega$ is only on diagonals while $\vec{k}$ is in every element of the matrix. However, the situation is completely different in the frequency-dependent case. Both $\vec{k}$ and $\omega$ exist in every element of the matrix. One may solve $\vec{k}$ for the input values of $\omega$, as well as determining the $\omega$ values entering the $\vec{k}$ as input. The two methods reveal different physical pictures [15].

Choosing real $\omega$ values as input, one determines, in general, complex $\vec{k}$ values whose imaginary part gives the spatial attenuation of the propagating wave. On the other hand, on entering real $\vec{k}$ values, one solves for complex $\omega$, whose imaginary part determines the temporal attenuation of the wave. Since we are mainly interested in the spatial attenuation of the waves, we followed the first method. However, we checked that the two approaches give parallel results. As a result of this procedure, we obtain complex wave vector values. We denote the real and imaginary parts of the wave vector as $k=k_{R}+i k_{I}$.

We note that the $k_{I}$ value may imply two different phenomena: reflection or absorption. If the dielectric function is real, the imaginary part of the wave vector, $k_{I}$, has a simple interpretation. The incident wave is totally reflected while penetrating into the crystal up to a distance of $2 \pi / k_{I}$. However, if the dielectric function is complex one cannot distinguish between reflection and absorption for a given value of $k_{I}$. A mixture of both occurs.

In our computations, we use an $11 \times 11$ parallelogramshaped grid of plane waves. This corresponds to a $(121 \times 121)$-dimensional matrix of elements given in $\mathrm{Eq}$. (13). We define the determinant of the matrix as a function of $\vec{k}$ and solve for the zeros of this complex function. We determined the complex $\vec{k}$ values corresponding to real $\omega$. The resulting band structures are plotted in Figs. 4 and 5.

Although we described our method for the TE modes, TM modes can be calculated similarly.

\section{RESULTS AND DISCUSSION}

When the dielectric function is frequency independent, the master equation (8) is scalable. That is, the structure of photonic bands, expressed in terms of the scaled frequency $\omega^{\prime}=\omega a / 2 \pi c$, is independent of the dimensions of the unit cell. It depends only on the lattice type and the internal struc- 

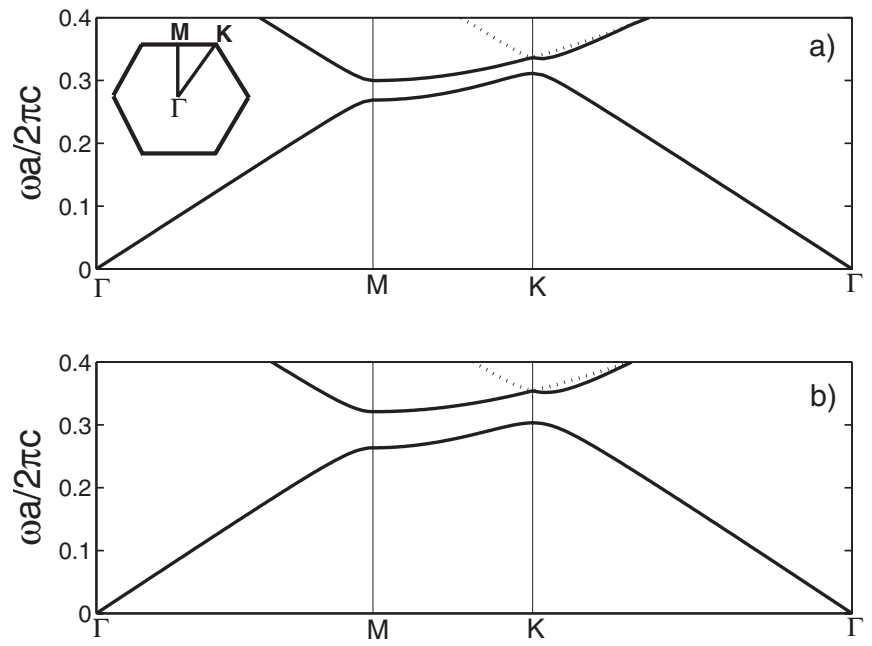

FIG. 3. TE modes of a triangular vortex lattice with frequencyindependent $\epsilon$. (Symmetry points and the irreducible Brillouin zone of a triangular lattice are indicated in the inset.) Dielectric constants and lattice parameters are (a) $\epsilon=5.2$ and $a=10 \xi$, (b) $\epsilon=8$ and $a=4.5 \xi$. Filling fractions of vortices, $f=(2 \pi / \sqrt{3}) \times\left(R^{2} / a^{2}\right)$ with effective radius $R \simeq 2 \xi$ are $15 \%$ and $71 \%$, respectively. Dielectric constant is the value of dielectric function (5) at the enhancement frequency, $\epsilon=\epsilon_{\mathrm{loc}}\left(\varpi_{0}\right)$. Density profile of the unit cell is treated using the Padé approximation [24]. (a) There exists a directional pseudo band gap with midgap frequency at $\omega_{g}^{\prime}=0.285$. (b) There is a complete band gap with gap center at $\omega_{g}^{\prime}=0.31$.

ture within the unit cell. On the other hand, such a scaling is not possible in the case of a frequency-dependent dielectric; since a new length scale is introduced into the system. Thus, we first calculate the band structure with constant $\epsilon$, and then discuss the change due to the frequency dependence of the dielectric constant.

We give the constant dielectric photonic bands of a triangular lattice of rubidium gas for two different sets of parameters in Fig. 3. The first set, $\Gamma M$ and $a=10 \xi$, has a directional pseudogap in the $\Gamma M$ direction, Fig. 3(a), when the dielectric constant is chosen as its value at the enhancement frequency, $\epsilon=\epsilon_{\mathrm{loc}}\left(\varpi_{0}\right)=5.2$. The pseudogap lies in the frequency range $\omega=(0.27-0.31)(2 \pi c / a)$, with its center at $\omega_{g}$ $=0.285(2 \pi c / a)$.

The second set of parameters, $N=6.6 \times 10^{20} \mathrm{~m}^{-3}$ and $a$ $=4.5 \xi$, is chosen so that there is a complete photonic band gap when the dielectric constant is at its enhanced value $\epsilon$ $=\epsilon_{\text {loc }}\left(\varpi_{0}\right)=8.0$. The band gap lies in the range $\omega$ $=(0.30-0.32)(2 \pi c / a)$ with midgap frequency $M K$.

Strong frequency dependence of the dielectric susceptibility, Fig. 2, will modify the structure of the bands significantly. We note that the dielectric function (5) differs from the vacuum value (unity) only in the frequency range of $\omega=\Omega_{0} \pm 5 \gamma$. The natural lattice frequency $2 \pi c / a$ that we used in the scaling of Fig. 3 is seven orders of magnitude greater than the decay rate $\gamma$. For typical values of the lattice parameter in a rotating BEC, $a \sim 200 \mathrm{~nm}$, the lattice frequency is $2 \pi c / a \sim 10^{15} \mathrm{~Hz}$, whereas the decay rate is only $\gamma=2 \pi \times 6 \times 10^{6} \mathrm{~Hz}$. The bands of frequency-dependent $\epsilon_{\text {loc }}(\omega)$ will be different from the propagation in vacuum, for only about $\sim 10 \gamma$ around the enhancement frequency $\Omega_{0}$. On the other hand, index enhancement without absorption is achievable in a narrower range of frequency, about $0.1 \gamma$.

Still, the constant $\epsilon$ bands give us an idea about how to arrange the lattice parameter $a$ to obtain a band gap with the frequency-dependent $\epsilon_{\mathrm{loc}}(\omega)$. In order to obtain a gap, we must arrange the enhancement frequency $\Omega_{0}$ such that it lies in the band gap of the corresponding constant dielectric case. A good choice is to place $\Omega_{0}$ at the center of the band gap, $\omega_{g}$. Thus, we tune the lattice parameter $a$ such that $\Omega_{0}=\omega_{g}^{\prime}(2 \pi c / a)$, which gives

$$
a=\omega_{g}^{\prime} \frac{2 \pi c}{\omega_{a b}+\varpi_{0} \gamma},
$$

where $\omega_{g}^{\prime}$ is obtained from constant dielectric calculations as in Fig. 3.

In conventional photonic crystals, it is generally not possible to change the lattice parameter, once the sample is manufactured. However, in the case of a rotating BEC, spacing between the vortex cores is continuously tunable. The density of the vortices depends on the rotation frequency, so the lattice parameter $a$ can be decreased (increased) by increasing (decreasing) the rotation rate. The filling factor $f$ of the lattice depends on $f \sim(\xi / a)^{2}$, as the coherence length $\xi$ determines the vortex core radius [see Eq. (7)]. The coherence length $\xi$ can be adjusted by the density $N$. Alternatively, $\xi$ can be adjusted by controlling $a_{\mathrm{sc}}$ via Feshbach resonances [26]. We note that one might be able to design more convenient sets of parameters for specific experiments. Using different alkali-metal atoms, like cesium, stronger index contrasts can be achieved due to the larger transition wavelengths. By employing different index enhancement schemes, such as the Raman scheme, broader index enhancement windows could translate to wider band gaps.

Choosing the lattice parameter $a$ as in Eq. (17), we calculate the photonic bands for the frequency-dependent dielectric function (6). Away from the enhancement frequency $\varpi_{0}$, the dielectric function is complex [Eqs. (1) and (2)]. Band structures are depicted in Figs. 4 and 5 for the same density and filling factor parameters as in Figs. 3(a) and 3(b), respectively. In both Figs. 4 and 5 the real and the imaginary parts of the wave vector, $k_{R}$ and $k_{I}$, are displayed separately. The enhancement frequency $\varpi_{0}=1.22$ is marked in all plots. The lattice parameters $a$ are chosen as $a=226 \mathrm{~nm}$ in Fig. 4 and $a=246 \mathrm{~nm}$ in Fig. 5 .

For a real dielectric function, it is very easy to identify the band gaps. The wave vector $k$ is real when there is propagation, and complex (with $k_{R}$ on the band edge) if the frequency is in a band gap. For a complex $\epsilon(\omega)$, however, identification of band gaps is not straightforward. One can determine the existence of a band gap by considering the frequency values where $\epsilon$ is real. If a nonzero $k_{I}$ is present, then there exists a band gap at that frequency. However, the width of the band gap cannot be directly identified by considering only the $k_{I}$ values away from the enhancement frequency. For a complex $\epsilon$, a nonzero value of $k_{I}$ may be due to the absorption as well as the effect of the band gap. Thus, we first discuss the existence of the band gaps in Figs. 4 and 5 and discuss the gap widths later. 

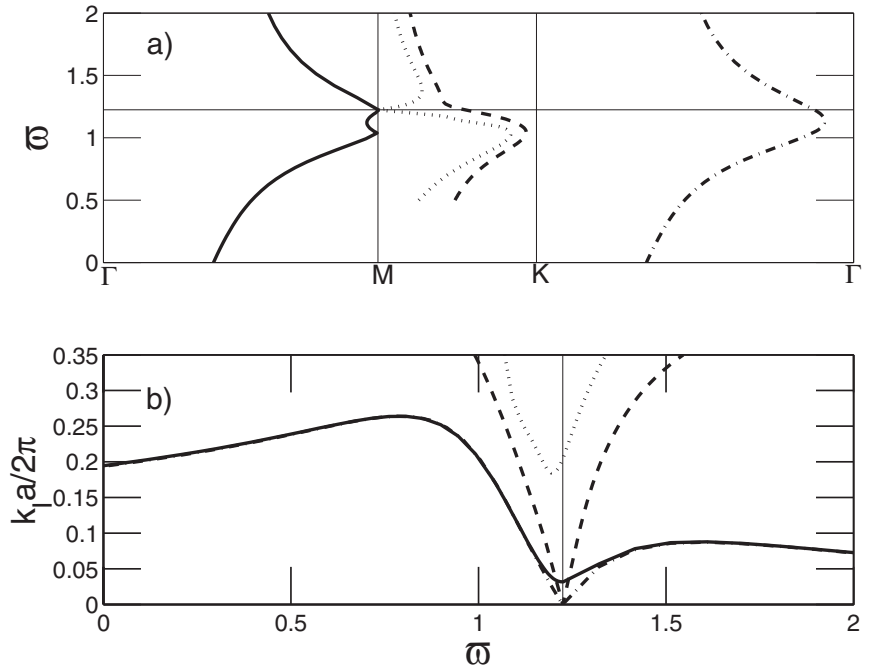

FIG. 4. (a) TE modes of triangular vortex lattice with frequency dependent dielectric function $\epsilon_{\text {loc }}(\varpi)$ (Fig. 2), and (b) imaginary parts of the wave vector $k_{I}$ corresponding to each mode. Particle density is $N=5.5 \times 10^{20} \mathrm{~m}^{-3}$ and lattice constant is $a=10 \xi$. Enhancement frequency $\Omega_{0}$ is tuned to the band gap at the $M$ edge $\left[\omega_{g}=0.285(2 \pi c / a)\right]$ of the constant dielectric case [Fig. 3(a) ]. $M K$ bands are plotted in a limited region, because of high $k_{I}$ values out of the given frequency region. There exists a directional gap in the $\Gamma M$ propagation direction.

In Fig. 4(b), the imaginary part of the wave vector $k_{I}$ is plotted for different propagation directions. In the $\Gamma_{M}$ and $\Gamma_{K}$ directions only a single band exists within the enhancement window, while for the $M K$ direction there are two bands. At the enhancement frequency $\varpi_{0}=1.22$, two of these bands have zero $k_{I}$, while the other two have a complex wave vector. In accordance with the discussion in the previous para-
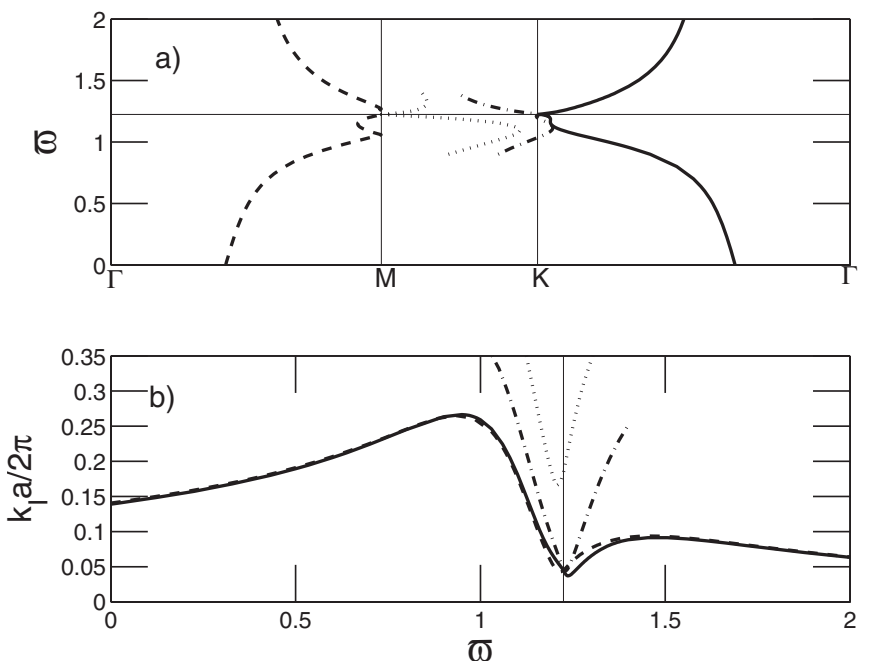

FIG. 5. (a) TE bands of triangular vortex lattice with frequencydependent dielectric function $\epsilon_{\mathrm{loc}}(\varpi)$ [Fig. 2(b)], and (b) imaginary parts of the wave vector $k_{I}$ corresponding to each mode. Particle density is $N=6.6 \times 10^{20} \mathrm{~m}^{-3}$ and lattice constant is $a=4.5 \xi$. Enhancement frequency $\Omega_{0}$ is tuned to the band gap at the $M$ edge $\left[\omega_{g}=0.31(2 \pi c / a)\right]$ of the constant dielectric case [Fig. 3(b)]. There exists a complete band gap.

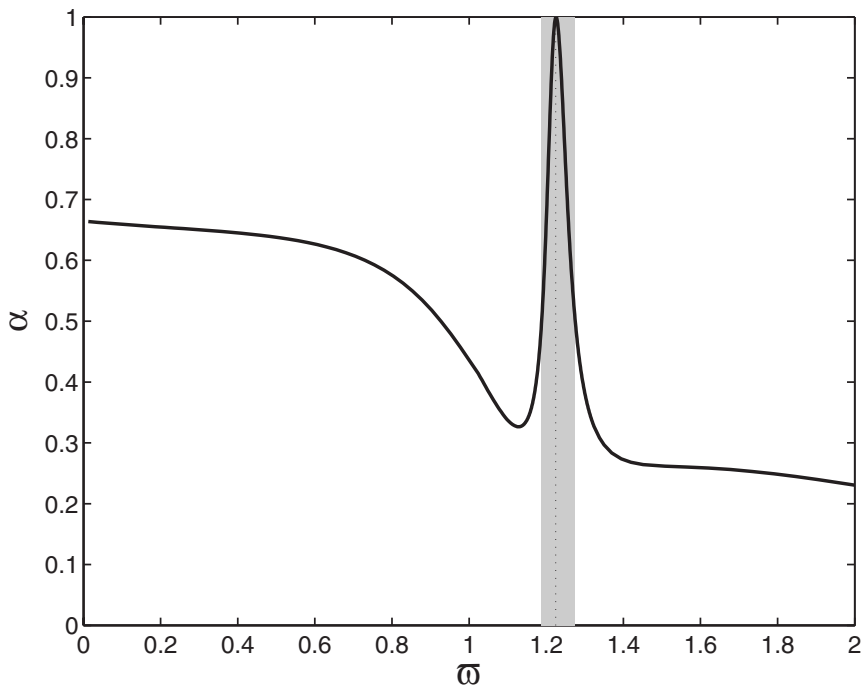

FIG. 6. Reactive energy ratio $\alpha$ for the $\Gamma M$ band of Fig. 4 . Vertical dashed line indicates the enhancement frequency $\varpi_{0}$ $=1.22$. Shaded region is the effective photonic band gap. Width of the peak determines the width of the gap to be $\omega=\Omega \pm 0.043 \gamma$ which corresponds to $\pm 1.65 \mathrm{MHz}$.

graph, we identify the existence of a pseudo band gap in the $\Gamma M$ propagation direction. Thus, incident light (exactly at $\left.\varpi_{0}\right)$ would propagate in the $\Gamma K$ and $M K$ directions while it would be stopped in the $\Gamma M$ direction.

In the second case, Fig. 5, all of the four bands have nonzero $k_{I}$ at $\varpi=\varpi_{0}$. This indicates the existence of a complete band gap at the enhancement frequency. Incident light is stopped for all propagation directions.

We see that the conclusions for the existence of photonic band gaps obtained by constant $\epsilon$ calculations are not modified, even when the strong frequency dependence of $\epsilon(\omega)$ is taken into account.

Although the existence of directional and complete band gaps is demonstrated at the enhancement frequency, the widths of these gaps cannot be determined by just investigating the behavior of $k_{I}$. In the vicinity of $\varpi=\varpi_{0}$, one cannot determine whether it is the absorption, or the existence of a band gap, that causes the decaying behavior of the wave $\left(e^{-\vec{k}_{r} \vec{r}}\right)$.

To be able to define the width of the gap, we calculate the behavior of the Poynting vector

$$
\vec{S}(\vec{r})=\frac{1}{2} \vec{E}(\vec{r}) \times \vec{H}(\vec{r})^{*}
$$

in the crystal. The real part of the Poynting vector, $\vec{S}_{R}(\vec{r})$, gives the energy flux of the field at position $\vec{r}$. The imaginary part $\vec{S}_{I}(\vec{r})$ is a measure of the reactive (stored) energy [27]. For a frequency-dependent, but real, $\epsilon(\omega)$, the Poynting vector is purely imaginary in the band gaps and real otherwise. However, for complex $\epsilon(\omega)$, the imaginary part of the $\vec{S}$ may also be due to absorption. Although we make similar statements about $\vec{k}_{I}$ and $\vec{S}_{I}$, together they are sufficient to determine the width of the band gap. 
Using the results of our band structure calculations, we compute the average of the Poynting vector, $\langle\vec{S}\rangle$, in the unit cell. We define

$$
\alpha=\left|\left\langle S_{I}\right\rangle\right| /|\langle S\rangle|
$$

which corresponds to the rate of reactive energy. The averages of $\left\langle S_{I}\right\rangle$ and $\langle S\rangle$ are computed along the $\Gamma M$ direction in order to investigate the gap width in Fig. 4.

Figure 6 displays a marked increase in the reactive energy ratio near the enhancement frequency. This increase cannot be directly caused by the imaginary part of $\epsilon_{\mathrm{loc}}(\varpi)$, as near the enhancement frequency this imaginary part is decreasing to zero. Thus, the peak in the reactive energy ratio must be caused mainly by the periodicity of the crystal. The presence of the band gap increases the reactive energy ratio despite decreasing absorption.

We define the width of the photonic band gap as the full width at half maximum of the peak at the enhancement frequency. With this definition we find an effective gap in the frequency range $\varpi=\varpi_{0} \pm 0.043$. In familiar units this translates to a bandwidth of $3.30 \mathrm{MHz}$. Using the same method we find a band gap of width $5.98 \mathrm{MHz}$ for the parameters of Fig. 5.

We also performed similar calculations for the TM modes and obtained similar band structures. The TM modes also give directional and complete band gaps when the lattice parameter is properly tuned. However, since the band gaps of TE and TM modes do not coincide in general, one cannot obtain band gaps for both modes, without further tuning.

\section{CONCLUSION}

We calculated the photonic bands for an index-enhanced vortex lattice, considering a frequency-dependent dielectric function. Our motivation was the possibility of the direct measurement of the rotation frequency in Bose-Einstein condensates using the directional band gap of the photonic crystal. We validated the main conclusion of our previous work [9], that photonic band gaps can be created via index enhancement on vortex lattices of BECs. Specifically, we presented two examples showing that both directional and complete band gaps are possible within experimentally realizable parameter regimes. For the specific parameters and the index enhancement scheme we considered, band gaps of order a few megahertz width are obtained. We also discussed how band gaps are designed for specific parameter values, and how band gap widths can be increased.

Unlike the previous results for metallic photonic crystals, here the complex dielectric function varies rapidly with the frequency for index-enhanced media. The strong frequency dependence is due to the high dipole moment, established through atomic coherence in a narrow frequency range. To our knowledge, such a periodic structure, composed of index-enhanced media, is here investigated for the first time. We showed that the photonic band structure in such a medium can be reliably calculated by numerically computing the zeros of the determinant of the master equation. We also developed a method, based on the calculation of the Poynting vector, to determine the effective widths of the photonic band gaps in media with frequency-dependent complex dielectric functions.

Ö.E.M. acknowledges support from a TÜBA/GEBİP grant. M.Ö.O. is supported by a TÜBA/GEBIP grant and TÜBITTAK-KARIYYER Grant No. 104T165.
[1] J. R. Abo-Shaeer, C. Raman, J. M. Vogels, and W. Ketterle, Science 292, 476 (2001).

[2] P. C. Haljan, I. Coddington, P. Engels, and E. A. Cornell, Phys. Rev. Lett. 87, 210403 (2001).

[3] T.-L. Ho, Phys. Rev. Lett. 87, 060403 (2001).

[4] G. Baym and C. J. Pethick, Phys. Rev. A 69, 043619 (2004).

[5] K. W. Madison, F. Chevy, W. Wohlleben, and J. Dalibard, Phys. Rev. Lett. 84, 806 (2000).

[6] K. W. Madison, F. Chevy, V. Bretin, and J. Dalibard, Phys. Rev. Lett. 86, 4443 (2001).

[7] N. L. Smith, W. H. Heathcote, J. M. Krueger, and C. J. Foot, Phys. Rev. Lett. 93, 080406 (2004).

[8] P. Engels, I. Coddington, P. C. Haljan, and E. A. Cornell, Phys. Rev. Lett. 89, 100403 (2002).

[9] Ö. E. Müstecaplıglu and M. Ö. Oktel, Phys. Rev. Lett. 94, 220404 (2005).

[10] M. Fleischhauer, C. H. Keitel, M. O. Scully, C. Su, B. T. Ulrich, and S. Y. Zhu, Phys. Rev. A 46, 1468 (1992).

[11] M. O. Scully and M. S. Zubairy, Quantum Optics (Cambridge University Press, Cambridge, U.K., 1997).

[12] J. D. Joannopoulos, R. D. Meade, and J. N. Winn, Photonic Crystals: Molding the Flow of Light (Princeton University
Press, Princeton, NJ, 1995).

[13] A. A. Krokhin and P. Halevi, Phys. Rev. B 53, 1205 (1996).

[14] M. M. Sigalas, C. M. Soukoulis, C. T. Chan, and K. M. Ho, Phys. Rev. B 49, 11080 (1994).

[15] V. Kuzmiak and A. A. Maradudin, Phys. Rev. B 55, 7427 (1997).

[16] L.-M. Li, Z.-Q. Zhang, and X. Zhang, Phys. Rev. B 58, 15589 (1998).

[17] A. Moroz, A. Tip, and J.-M. Combes, Synth. Met. 116, 481 (2001).

[18] A. Tip, A. Moroz, and J. M. Combes, J. Phys. A 33, 6223 (2000).

[19] H. van der Lem, A. Tip, and A. Moroz, J. Opt. Soc. Am. B 20, 1334 (2003).0740-3224

[20] G. Veronis, R. W. Dutton, and S. Fan, J. Appl. Phys. 97, 093104 (2005).

[21] X. Jiang and C. M. Soukoulis, Phys. Rev. B 59, 6159 (1999).

[22] Y. V. Rostovtsev, A. B. Matsko, and M. O. Scully, Phys. Rev. A 57, 4919 (1998).

[23] J. Ruostekoski and J. Javanainen, Phys. Rev. Lett. 82, 4741 (1999); M. Fleischhauer, Phys. Rev. A 60, 2534 (1999); H. Wallis, ibid. 56, 2060 (1997); O. Morice, Y. Castin, and J. 
Dalibard, ibid. 51, 3896 (1995); J. Ruostekoski and J. Javanainen, ibid. 56, 2056 (1997); K. V. Krutitsky, K.-P. Marzlin, and J. Audretsch, ibid. 65, 063609 (2002).

[24] N. G. Berloff, J. Phys. A 37, 1617 (2004).

[25] A. Tip, A. Moroz, and J. M. Combes, J. Phys. A 33, 6223 (2000); C. Kittel, Introduction to Solid State Physics (Wiley,
New York, 1996).

[26] S. L. Cornish, N. R. Claussen, J. L. Roberts, E. A. Cornell, and C. E. Wieman, Phys. Rev. Lett. 85, 1795 (2000).

[27] J. D. Jackson, Classical Electrodynamics (Wiley, New York, 1999). 